\title{
Partial and Semi-Partial Correlations for Categorical Variables in Educational Research: Addressing Two Common Misconceptions
}

\begin{tabular}{cc}
\hline Hongwei Yang & Wing Hang Wong \\
Arizona State University & University of Kentucky \\
Kelly D. Bradley & Michael D. Toland \\
University of Kentucky & University of Kentucky \\
\hline \hline
\end{tabular}

This article addresses two misconceptions that educational researchers have about partial and semi-partial correlations. To undo the two misconceptions, this article provides a detailed discussion of the two types of correlations under linear regression which includes multiple dummy indicator variables created for a categorical predictor. A numerical example is provided to demonstrate how the two correlations can be properly utilized to solve research questions in education: 1) Interpretation under the substantive problem and 2) assessment of relative importance of predictors in predicting the dependent variable (DV). The article concludes with a summary of findings and provides the SPSS code used so that interested readers may replicate the results.

$\mathbf{P}$ artial and semi-partial correlations (a.k.a., part correlations) have had a long history of being used to address research questions in education (Ary, Jacobs, Sorensen, \& Walker, 2013; Johnson, \& Christensen, 2010; Ley, 1972; Pedhazur, \& Schmelkin, 1991; Phelps, 1923; Sachar, 1980). Many research questions in education can be analyzed under these two correlations. For example, it may be that ACT scores can be used to predict college GPA because those test scores provide much of the same information given by another excellent predictor, high school GPA. Then, the question might be asked, "Do ACT scores still predict college GPA if we look only at students with the same high school GPA?" Stated differently, the question is, "What would the relationship between ACT scores and college GPA have been if each student had the same high school GPA?" To answer this question, we need the partial correlation coefficient between college GPA and ACT scores that adjusts for high school GPA. Here, high school GPA has been held constant and thus removed as a source of variation from both college GPA and ACT scores. In another case, we may ask a similar, but different question, "Given that a correlation exists between high school GPA and ACT scores, suppose that the ACT scores have been adjusted to remove the influence of high school performance. How well would the adjusted ACT scores then correlate with college GPA?" This second question would be investigated using the semi-partial correlation between college GPA and adjusted ACT scores, in which the effect of high school GPA has been partialled out from the ACT scores, but not from the DV (college GPA).

In a real sense, the use of partial and semi-partial correlations is a statistical substitute for experimental controls. That is, both correlation coefficients are statistical control techniques that are able to mathematically partial out the (linear) effects of some other extraneous variables on one or both correlation variables. In the statistical literature, phrases such as "partial out", "control for", "adjust for", "hold constant", "correct for the influence of", and similar terms, are often used inter-changeably to refer to statistical control where control means the control of (removal of) variance. Statistical control serves to exert control over extraneous variables by applying the proper statistical or mathematical procedures, rather than through experimental procedures (Cohen, Cohen, West, \& Aiken, 2003). This control is particularly important for analyzing observational data, and is different from experimental control for experimental data where the researcher manipulates the nature of the sample or environment so that it is identical across participants (Keppel, 1991). With extraneous variables statistically controlled, the impression of observed correlations between variables may substantially change.

In linear regression, it is often necessary to hold constant the effects of other predictors when examining the relationship between one predictor and the DV. This is because predictors are related not only to the DV, but typically also to each other. The extent to which predictors are correlated with each other is an index of the overlap or redundancy of the information that they provide about the DV. In the college GPA example, one might just want to know how two predictor variables, high school GPA and ACT scores, predict the DV of college GPA after the redundant variance shared by the two predictors has been removed. This is more than an esoteric concern. Whenever test scores are used to make decisions 
Yang et al.

about screening, placement or selection, as a policy matter, it is important to know which scores should be more heavily weighted in the decisions. This can be achieved by removing the variance overlap or redundancy in predictors, as is the case with partial and semi-partial correlations for predictor variables

\section{Purpose of Study}

Despite the long-standing usefulness of the two types of correlations in education, a review of the literature indicates that there are misconceptions about them among educational researchers. At least two misconceptions exist, one associated with the partial correlation and the other with the semi-partial correlation:

1. Misconception 1: Partial correlations cannot be used for categorical variables. For example, Johnson and Christensen (2010, p. 358) argue that all variables used in partial correlation analysis must be quantitative rather than categorical. Price (n.d.) and Psychwiki. (n.d.) say that all variables (DV, predictor variable being correlated with the DV, and predictor variable(s) being controlled for) used in computing partial correlations have to be continuous.

2. Misconception 2: Semi-partial correlations are semi- in the sense that only half of the other predictors are being controlled for. For example, Martella, Nelson, Morgan, and MarchandMartella (2013, p. 221) argue that the semi-partial correlation between the DV and a predictor can be calculated when either one of the two remaining predictors (but not both) is being controlled for. Oddly, the authors correctly describe the theoretical implication of the semipartial correlation elsewhere in the book, but they fail to translate that theoretical description into a solution to the concrete example.

The two misconceptions are very unfortunate. Whereas the opinion in misconception 1 may be just inaccurate or misleading, if not totally incorrect, that in misconception 2 is simply not true. Revision of misconception 1 would state that partial correlations can be used for categorical predictor variables as long as they are properly coded. Here, a categorical predictor could be either the variable that is being correlated with DV or the variable that is being controlled for. Revision of misconception 2 would state that semi-partial correlations serve to control for the effects of the remaining predictors on only the predictor being correlated with the DV (without removing their effects on the DV), instead of controlling for half of the remaining predictors. Next, this article works to undo these two misconceptions.

We conducted an extensive review of the literature on correlation, regression, and linear models, only to find very few authors discuss the two types of correlations for categorical predictors (Draper \& Smith, 1981; Edwards, 1976; Gelman \& Hill, 2007; Hays, 1994; Kutner, Nachtsheim, Neter, \& Li, 2005; LewisBeck, 1980; Mendenhall \& Sincich, 2003; Monahan, 2008; Montgomery, Peck, \& Vining, 2001; Muller \& Fetterman, 2002; Neter \& Wasserman, 1974; Rao \& Toutenburg, 1999; Searle, 1971; Seber \& Lee, 2003; Weisberg, 2005; Yan \& Su, 2009), although many of them cover the correlations for quantitative variables. Cohen et al. (2003) and Field (2009) are two of the few books that provide a dedicated discussion on partial and semi-partial correlations for categorical predictors with the latter coverage being very limited. Therefore, this article builds upon the work of Cohen et al.

\section{Computation Procedures for Partial and Semi-Partial Correlations}

We begin with a demonstration of the computation procedure of each correlation in a linear regression model where there are four predictors. So, each time a (partial/semi-partial) correlation is computed for a predictor (with the DV), the number of variables controlled for is three. Given three predictors to be partialled out, the partial and semi-partial correlations are both third-order. More generally, given a certain number of predictors, all but one must be controlled for when correlating one predictor with the DV. Because the order of a correlation coefficient equals the number of controlled variables, a fourth-order partial/semi-partial correlation is defined when we account for the effects of four predictors. Similarly, we can define higher/lower-order partial and semi-partial correlations. Accordingly, given no variables to control for, the correlation coefficient is called a zero-order correlation. Many times, we further square partial and semi-partial correlations to obtain coefficients of partial and semi-partial determination, which is much like squaring the coefficient of multiple correlation to obtain the coefficient of multiple determination ( $R^{2}$ statistic).

A linear regression model containing four predictors is defined as follows:

$$
Y_{i}=\beta_{0}+\beta_{1} X_{1 i}+\beta_{2} X_{2 i}+\beta_{3} X_{3 i}+\beta_{4} X_{4 i}+\varepsilon_{i},
$$


where $i=1,2, \ldots n$, and $n$ is the sample size. In Equation 1, partial and semi-partial correlations can be calculated for any of the four predictors: $X_{1}, X_{2}, X_{3}$, and $X_{4}$. We take $X_{1}$ as an example without losing generalizability. When computing the two correlations for $X_{1}$, we account for the effects of $X_{2}, X_{3}$, and $X_{4}$ combined. As for the notation, we use $r_{Y X_{1} X_{2} X_{3} X_{4}}$ to represent the partial correlation between $Y$ and $X_{1}$, and use $r_{Y\left(X_{1}, X_{2} X_{3} X_{4}\right)}$ to represent the semi-partial correlation. In both cases, the dot notation in subscripts indicates what is being partialled from what: $Y X_{1} \cdot X_{2} X_{3} X_{4}$ means $X_{2}, X_{3}$, and $X_{4}$ combined are partialled from both $Y$ and $X_{1}$, whereas $Y\left(X_{1} \cdot X_{2} X_{3} X_{4}\right)$ means $X_{2}, X_{3}$, and $X_{4}$ combined are partialled from only $X_{1}$. We use $R_{Y . X_{1} X_{2} X_{3} X_{4}}^{2}$ to represent the coefficient of multiple determination $\left(R^{2}\right.$ statistic $)$ when regressing $Y$ on all four predictors. Finally, we use $r_{Y X_{1}}$ to indicate the zero-order correlation between $Y$ and $X_{1}$.

Next, we present the two types of correlations through partitioning of the total sum of squares of the DV into various sources, an extra sum of squares method designed to measure the marginal reduction in the error sum of squares when one or several predictor variables are added to the regression model, given certain predictor variables already in the model. These sum of squares statistics are typically available in regression outputs and are easy to understand.

Partial Correlation between $Y$ and $X_{1}$

The partial correlation $r_{Y X_{1} X_{2} X_{3} X_{4}}$ measures the correlation between $X_{1}$ and $Y$ when the linear effects of $X_{2}, X_{3}$, and $X_{4}$ have been removed from both. We first present the formula for the coefficient of partial determination $r_{Y X_{1}, X_{2} X_{3} X_{4}}^{2}$ before discussing the formula for partial correlation. According to Kutner et al. (2005) and Tamhane and Dunlop (2000), $r_{Y X_{1}, X_{2} X_{3} X_{4}}^{2}$ is given by

$$
r_{Y X_{1}, X_{2} X_{3} X_{4}}^{2}=\frac{S S R\left(X_{1} \mid X_{2}, X_{3}, X_{4}\right)}{S S E\left(X_{2}, X_{3}, X_{4}\right)}=\frac{\operatorname{SSE}\left(X_{2}, X_{3}, X_{4}\right)-S S E\left(X_{1}, X_{2}, X_{3}, X_{4}\right)}{S S E\left(X_{2}, X_{3}, X_{4}\right)} ;
$$

where $\operatorname{SSR}\left(X_{1} \mid X_{2}, X_{3}, X_{4}\right)$ is the extra sum of squares statistic measuring the marginal reduction in the error sum of squares when $X_{1}$ is added to the regression model that has already had $X_{2}, X_{3}$, and $X_{4}$ in it, $\operatorname{SSE}\left(X_{1}, X_{2}, X_{3}, X_{4}\right)$ and $\operatorname{SSE}\left(X_{2}, X_{3}, X_{4}\right)$ are, respectively, the error sum of squares from the regression of $Y$ on all four predictors, and on just three of them: $X_{2}, X_{3}$, and $X_{4}$. With $r_{Y X_{1} \cdot X_{2} X_{3} X_{4}}^{2}$ defined, the partial correlation $r_{Y X_{1} . X_{2} X_{3} X_{4}}$ is simply the square root of $r_{Y X_{1} X_{2} X_{3} X_{4}}^{2}$, and it is given the same sign as that of the corresponding regression coefficient in the fitted regression model.

An interpretation of the coefficient of partial determination can be obtained from its definition in Equation 2. The numerator of the equation is the reduction in the error sum of squares when $X_{1}$ is added to the regression model which already has $X_{2}, X_{3}$, and $X_{4}$ in it. In other words, it represents the amount of variability in $Y$ that $X_{1}$ uniquely explains beyond the amount already explained by an optimally weighted linear combination of $X_{2}, X_{3}$, and $X_{4}$. The denominator of the equation is the error sum of squares when the model contains only $X_{2}, X_{3}$, and $X_{4}$. In other words, it represents the amount of variability in $Y$ that an optimally weighted linear combination of $X_{2}, X_{3}$, and $X_{4}$ fails to explain. So, the coefficient of partial determination for $X_{1}$ as defined in Equation 2 represents the proportion of the remaining DV variability left unexplained by $X_{2}, X_{3}$, and $X_{4}$ combined that is uniquely explained by $X_{1}$.

Next, the coefficient of partial correlation can also be calculated from the perspective of effects removed. Here, removing the effects of a set of variables (say, $X_{2}, X_{3}$, and $X_{4}$ combined) on another variable (say, $X_{1}$ ) is equivalent to subtracting from $X_{1}$ the values of $X_{1}$ predicted from an optimally weighted linear combination of $X_{2}, X_{3}$, and $X_{4}$. That is, to remove the effects of $X_{2}, X_{3}$, and $X_{4}$ on each of $Y$ and $X_{1}$, we have to calculate the residuals from regressing separately each variable $\left(Y\right.$ and $\left.X_{1}\right)$ on $X_{2}$, $X_{3}$, and $X_{4}$ combined. Next, the zero-order Pearson correlation between the two sets of residuals equals the partial correlation between $Y$ and $X_{1}$ after adjusting for their linear relationships to $X_{2}, X_{3}$, and $X_{4}$. As such, the coefficient of partial determination for $X_{1}$ can also be calculated as the coefficient of (simple) determination from squaring the zero-order correlation between the two sets of residuals (Draper, \& Smith, 1981; Hays, 1994; Kutner et al., 2005; Montgomery et al., 2001).

\section{Semi-Partial Correlation between $Y$ and $X_{1}$}

The semi-partial correlation $r_{Y\left(X_{1}, X_{2} X_{3} X_{4}\right)}$ measures the correlation between $X_{1}$ and $Y$ when the linear effects of $X_{2}, X_{3}$, and $X_{4}$ have been removed from only $X_{1}$. After squaring the semi-partial correlation coefficient, we obtain $r_{Y\left(X_{1}, X_{2} X_{3} X_{4}\right)}^{2}$ which measures the proportion of the total DV variability accounted 
Yang et al.

for by $X_{1}$ beyond the proportion already explained by the other three predictors $X_{2}, X_{3}$, and $X_{4}$ combined. Simply put, $r_{Y\left(X_{1} \cdot X_{2} X_{3} X_{4}\right)}^{2}$ equals the increase in the $R^{2}$ statistic when $X_{1}$ is added to a model that has already had $X_{2}, X_{3}$, and $X_{4}$ in it. Therefore, $r_{Y\left(X_{1} \cdot X_{2} X_{3} X_{4}\right)}^{2}$ represents the unique contribution of $X_{1}$ to the coefficient of multiple determination in the context of $X_{2}, X_{3}$, and $X_{4}$. So, according to Cohen et al. (2003), $r_{Y\left(X_{1}, X_{2} X_{3} X_{4}\right)}^{2}$ is given by

$$
r_{Y\left(X_{1} . X_{2} X_{3} X_{4}\right)}^{2}=\frac{S S R\left(X_{1} \mid X_{2}, X_{3}, X_{4}\right)}{S S T_{Y}}=\frac{S S E\left(X_{2}, X_{3}, X_{4}\right)-S S E\left(X_{1}, X_{2}, X_{3}, X_{4}\right)}{S S T_{Y}} ;
$$

where $S S T_{Y}$ is total sum of squares for the DV, and $\operatorname{SSR}\left(X_{1} \mid X_{2}, X_{3}, X_{4}\right), \operatorname{SSE}\left(X_{1}, X_{2}, X_{3}, X_{4}\right)$, and $\operatorname{SSE}\left(X_{2}, X_{3}, X_{4}\right)$ have been previously defined in Equation 2. Next, the semi-partial correlation $r_{Y\left(X_{1} X_{2} X_{3} X_{4}\right)}$ is simply the square root of $r_{Y\left(X_{1} \cdot X_{2} X_{3} X_{4}\right)}^{2}$, and it is given the same sign as that of the corresponding regression coefficient in the fitted regression model.

An interpretation of the coefficient of semi-partial determination can be obtained from its definition in Equation 3. The numerator of the equation is the reduction in the error sum of squares when $X_{1}$ is added to the regression model which already has $X_{2}, X_{3}$, and $X_{4}$ in it. In other words, it represents the amount of variability in $Y$ that $X_{1}$ uniquely explains beyond the amount already explained by an optimally weighted linear combination of $X_{2}, X_{3}$, and $X_{4}$. The denominator of the equation is the total sum of squares of the DV; it represents the total amount of variability in $Y$. So, the coefficient of semi-partial determination for $X_{1}$ as defined in Equation 3 represents the proportion of the total DV variability that is uniquely explained by $X_{1}$.

Next, the coefficient of semi-partial correlation can also be calculated from the perspective of effects removed. To that end, we calculate the set of residuals from regressing $X_{1}$ on $X_{2}, X_{3}$, and $X_{4}$ combined. Then, the zero-order Pearson correlation between the DV $(Y)$ and the calculated set of residuals equals the semi-partial correlation between $Y$ and $X_{1}$ after adjusting for the linear relationship of $X_{1}$ to $X_{2}, X_{3}$, and $X_{4}$ combined. Next, the coefficient of semi-partial determination for $X_{1}$ can be calculated as the coefficient of (simple) determination from squaring the zero-order correlation between $Y$ and the set of residuals from the regression of $X_{1}$ on $X_{2}, X_{3}$, and $X_{4}$ combined.

\section{Given Categorical Predictor Variables Correlated with the DV}

The definitions of partial and semi-partial correlations do not exclude categorical predictors, and the calculations involving one or more such variables are conducted in a similar way (Cohen et al., 2003; Field, 2009). Here, a categorical predictor variable could be one for which a partial/semi-partial correlation coefficient is being computed, and/or one whose effect is being adjusted for when calculating the two types of correlations for another predictor variable. Here, we primarily examine correlation coefficients computed for a categorical predictor, with a brief mention in the end of the case of a categorical predictor whose effect is controlled for.

A categorical predictor is typically numerically coded into contrast variables before it can be used in a regression model. One of the frequently used types of contrast variables is that of indicator variables or dummy variables. This dummy coding process uses one of the categories as the baseline of comparison (a.k.a., reference group/category where all dummy indicators assume the value of zero), and creates a dummy variable for each and every other category of the predictor. A dummy variable for a particular category is dichotomous which takes on only 1's and 0 's, with 1 representing the category is present for the observation and 0 absent for the observation. For a categorical predictor with $k$ categories, we need to create $(k-1)$ dummy variables to fully incorporate all categories of this predictor into a regression model that contains an intercept. Therefore, when examining the correlations between the DV and the categorical predictor, partial and semi-partial correlations are calculated for each of its dummy indicators.

For each dummy indicator, its partial correlation can be viewed as a representation of the corresponding unstandardized regression parameter estimate in a correlational rather than raw score metric (Cohen et al., 2003). Specifically, the partial correlation for a dummy predictor is an expression in correlational terms of the difference between the indicated group and the reference group in terms of the DV scores. By contrast, with the semi-partial correlation for a dummy indicator, it is usually its square that gets interpreted. The squared semi-partial correlation represents the reduction in the multiple 
coefficient of determination $\left(R^{2}\right.$ statistic) when the category indicated by this particular dummy variable is collapsed or combined with the baseline category of comparison (a.k.a., removing the distinction between this category and the reference category to come up with a combined, new category). In other words, the squared semi-partial correlation for a dummy variable provides a measure, in terms of the proportion of total DV variability, of the importance of distinguishing the group indicated by the dummy variable from the reference group where all dummy variables assume the value of zero.

Given that $X_{1}$ is one of the dichotomous dummy variables for a categorical predictor, Equation 2 can also be used to calculate its partial determination coefficient. When the categorical predictor has only two categories, $X_{1}$ is the only dummy variable that is needed to fully describe the categorical predictor. Equation 2 can be used to obtain the coefficient of partial determination for $X_{1}$ to adjust for $X_{2}, X_{3}$, and $X_{4}$ in the same manner as described above: Calculating the extra sum of squares statistic when $X_{1}$ is added to a model that already has $X_{2}, X_{3}$, and $X_{4}$ in it. Given that the categorical predictor has three categories, a second dummy variable $X_{2}$ is created which is in addition to $X_{1}$. In this case, to obtain the coefficient of partial determination for $X_{1}$, the extra sum of squares statistic has to be calculated for $X_{1}$ given the other dummy variable $X_{2}$ and any other predictors already in the model. Then Equation 2 can be used to calculate the partial determination coefficient for $X_{1}$. Next, the partial determination coefficient for $X_{2}$ can be obtained in a similar manner. This method is easily generalizable to a categorical predictor with an even larger number of categories. As long as we obtain the extra sum of squares statistic for each and every dummy variable given all other dummy variables and any other predictors in the model, we can easily obtain the coefficient of partial determination for that dummy variable which adjusts for everything else (all other dummy variables that belong to the same categorical predictor, and any other predictors), hence obtaining the partial correlation coefficient after computing the square root of the partial determination coefficient and taking into account the sign of the corresponding regression parameter estimate.

Given that $X_{1}$ is one of the dichotomous dummy variables for a categorical predictor, Equation 3 functions to compute its semi-partial determination coefficient in a manner similar to the way Equation 2 is used to compute its partial determination coefficient. As long as we are able to obtain the extra sum of squares statistic for that dummy variable given all other dummy variables (if any) and all other predictors (if any) in the model, we can easily obtain its coefficient of semi-partial determination which adjusts for everything else (any other dummy variables that belong to the same categorical predictor, and any other predictors), hence obtaining the semi-partial correlation coefficient after computing the square root of the semi-partial determination coefficient and taking into account the sign of the corresponding regression parameter estimate.

\section{A Numerical Example from Educational Research}

An example provided next demonstrates the use of partial and semi-partial correlations for dummy variables to address research questions in education. The artificial data set contains 232 complete observations taken from 232 first year college freshmen. Each student is measured on four variables: one DV and three predictors. A description of the variables is found below:

- Dependent variable: End-of-first-year college GPA

- Predictor variables:

- $X_{\text {Population }}$ - Population (ethnic) group each student belongs to: 1 for Asian, 2 for White, 3 for African American, and 4 for Others (Hispanics, American Indians, Alaskans, etc.)

- $X_{4}$ - Admission ACT score

- $X_{5}$ - High school GPA

Out of the three predictors here, $X_{4}$ (admission ACT score) and $X_{5}$ (high school GPA) are treated as continuous and $X_{\text {Population }}$ (population group) is categorical. Next, we proceed to create dummy variables for $X_{\text {Population. }}$ Given four categories, we need three dummy variables to fully incorporate this categorical predictor into a regression model that contains an intercept. Here, we choose the Others category $\left(X_{\text {Population }}=4\right.$ in the data set) as the reference level and create a dummy variable for each of the other three groups: 1) Dum1_Asian or $X_{1}$ for Asian, 2) Dum2_White or $X_{2}$ for White, and 3) Dum3_Africam or $X_{3}$ for African American. Then, the regression model is built that relates the DV to three dummy variables $\left(X_{1}, X_{2}\right.$, and $\left.X_{3}\right)$ and two continuous predictors $\left(X_{4}\right.$ and $\left.X_{5}\right)$. Finally, it should be noted that this example is 
Yang et al.

based on an artificial data set and is thus fictitious. Therefore, any resemblance to real research problems is purely coincidental.

To complete the (first) analysis, we use the REGRESSION command provided in SPSS 23. The command is run to fit two models. In model 1, students are analyzed in four groups (Asian, White, African American, and Others), whereas in model 2, the distinction between Asian and Others is eliminated (by removing Dum1_Asian from the model), which leads to three types of classifications (Others/Asian, White, and African American) with the new category of Others/Asian being the new reference level this time. That means, model 2 just uses two of the three dummy variables created above: 1) Dum2_White, and 2) Dum3_Africam. Dum1_Asian is not part of this second model because it is this dummy indicator that distinguishes Asian students from students in the Others group, given the distinctions made in the other two dummy variables. In other words, model 1 contains three dummy variables all of which assume the value of zero for the Others category, whereas model 2 contains only two dummy variables both of which assume the value of zero for the new Others/Asian category. Finally, we have the program also compute standardized parameter estimates and zero-order correlations for all predictors in the two models. Together with partial and semi-partial correlations, these additional statistics typically serve to evaluate the relative importance (to be defined later in the article) of predictors. The summary matrix (correlations, means, etc.) of the original data and the SPSS code used for the (first) analysis are provided in the appendix. A summary of model fit and inferential statistics information is found in Table 1.

A review of Table 1 indicates that both models provide a reasonable fit to the data with the coefficients of multiple determination being about $30.0 \%$ and the ANOVA tables (omitted from here) supporting a linear regression relationship between the DV and each set of predictors. Next, we primarily examine model 1: Its partial and semi-partial correlation/determination coefficients, along with some other statistics, for the three dummy indicators from the categorical predictor. During the process, we take $X_{1}$ as an example $\left(r_{Y X_{1} . X_{2} X_{3} X_{4} X_{5}}^{2}\right.$, etc.) and the conclusions generalize easily to other dummy predictors.

Table 1. Model Information from the SPSS REGRESSION Command

\begin{tabular}{|c|c|c|c|c|c|c|c|c|c|}
\hline \multirow[b]{2}{*}{$\begin{array}{c}\text { Model } \\
R^{2} \\
\end{array}$} & \multirow[b]{2}{*}{ Predictors } & \multicolumn{2}{|c|}{$\begin{array}{l}\text { Unstandardized } \\
\text { Coefficients }\end{array}$} & \multirow{2}{*}{$\frac{\begin{array}{c}\text { Standardize } \\
\text { Coefficients }\end{array}}{\text { Beta }}$} & \multirow{2}{*}{ d } & \multirow[b]{2}{*}{ Sig. } & \multicolumn{3}{|c|}{ Correlations } \\
\hline & & B & Std. Error & & & & $\begin{array}{l}\text { Zero- } \\
\text { Order } \\
\end{array}$ & Partial & Semi-Partial \\
\hline \multirow{6}{*}{$\begin{array}{c}1 \\
0.299\end{array}$} & (Constant) & -0.626 & 0.491 & & -1.275 & 0.204 & & & \\
\hline & dum1_asian & -1.160 & 0.474 & -0.173 & -2.449 & 0.015 & -0.132 & -0.161 & -0.136 \\
\hline & dum2_white & -0.189 & 0.300 & -0.067 & -0.629 & 0.530 & 0.148 & -0.042 & -0.035 \\
\hline & dum3_africam & -0.501 & 0.348 & -0.141 & -1.439 & 0.151 & -0.144 & -0.095 & -0.080 \\
\hline & e_act & 0.039 & 0.016 & 0.154 & 2.382 & 0.018 & 0.357 & 0.157 & 0.133 \\
\hline & hs_gpa & 0.767 & 0.115 & 0.422 & 6.656 & 0.000 & 0.501 & 0.405 & 0.371 \\
\hline \multirow{5}{*}{$\begin{array}{c}2 \\
0.281\end{array}$} & (Constant) & -1.025 & 0.468 & & -2.190 & 0.030 & & & \\
\hline & dum2_white & 0.263 & 0.239 & 0.093 & 1.099 & 0.273 & 0.148 & 0.073 & 0.062 \\
\hline & dum3_africam & -0.059 & 0.301 & -0.017 & -0.194 & 0.846 & -0.144 & -0.013 & -0.011 \\
\hline & e_act & 0.034 & 0.017 & 0.134 & 2.077 & 0.039 & 0.357 & 0.137 & 0.117 \\
\hline & hs_gpa & 0.787 & 0.116 & 0.432 & 6.771 & 0.000 & 0.501 & 0.410 & 0.381 \\
\hline
\end{tabular}

\section{Partial Correlations for Dummy Indicators}

As far as the partial correlation coefficient $r_{Y X_{1} . X_{2} X_{3} X_{4} X_{5}}=-0.161$ is concerned, it measures the relationship between $X_{1}$ and $Y$, after holding constant the effects on both of them from all other predictors. Given that $X_{1}$ for Asian students is one of the dummy indicators created for a categorical predictor, holding constant the other dummy variables means that it is within a subset of the data where the other two dummy indicators $\left(X_{2}\right.$ and $\left.X_{3}\right)$ remain invariant: Those cases that are neither White nor African 
American students. This subset of data is further restricted to those cases with identical values on $X_{4}$ and $X_{5}$. Therefore, we are left with students who are either Asian or Others, and are identical on admission ACT score and high school GPA. From here, we investigate the distinction between these two groups of students (Asian versus Others). Specifically, $r_{Y X_{1} X_{2} X_{3} X_{4} X_{5}}=-0.161$ in the current example is the correlation between college GPA (DV) and Asian versus non-Asian (a.k.a., Others), holding constant White versus non-White, African American versus non-African American, and finally the continuous

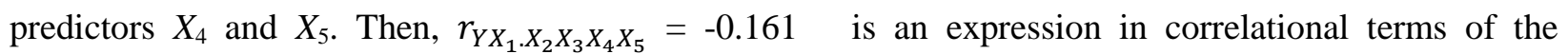
difference in college GPA (DV) between Asian students and students from the Others group, given the distinctions made in the other two dummy variables and given the two continuous predictors.

On the other hand, we can also square $r_{Y X_{1} . X_{2} X_{3} X_{4} X_{5}}$ and study the relationship from the perspective of the coefficient of partial determination using the extra sum of squares statistic. Given that $r_{Y X_{1} X_{2} X_{3} X_{4} X_{5}}^{2}=$ 0.026, we conclude that, within those students who are neither White nor African American and are identical on admission ACT score and high school GPA, a new distinction made between Asian students and other students (Others) is estimated to help the model explain about $2.6 \%$ of the remaining variability in the DV left unexplained by $X_{4}$ and $X_{5}$ and the classification of students into only three groups: White, African American, and Others/Asian. In other words, given that we continue to keep in the model the distinction between White and non-White, the distinction between African American and nonAfrican American, admission ACT score, and high school GPA, the removal of the distinction between Asian and other students (Others), which results in a new reference category of Others/Asian, would lead to an increase (in unexplained DV variability) of $2.669 \%=100[0.026 /(1-0.026)]$ of the amount of remaining DV variability left unexplained by a combination of the original categorical predictor with four groups of students and $X_{4}$ and $X_{5}$.

\section{Semi-Partial Correlations for Dummy Indicators}

As far as the semi-partial correlation coefficient $r_{Y\left(X_{1}, X_{2} X_{3} X_{4} X_{5}\right)}=-0.136$ is concerned, it measures the relationship between $X_{1}$ and $Y$, after holding constant the effects on only $X_{1}$ from all other predictors. Because its square or the coefficient of semi-partial determination is closely related to the commonly used $R^{2}$ statistic, we choose to discuss the $r_{Y\left(X_{1} X_{2} X_{3} X_{4} X_{5}\right)}^{2}$ statistic here which is more intuitive to understand. In this scenario, $r_{Y\left(X_{1} \cdot X_{2} X_{3} X_{4} X_{5}\right)}^{2}=R_{Y \cdot X_{1} X_{2} X_{3} X_{4} X_{5}}^{2}-R_{Y \cdot X_{2} X_{3} X_{4} X_{5}}^{2}$, and the absence of $X_{1}$ in the subscript of $R_{Y . X_{2} X_{3} X_{4} X_{5}}^{2}$ means collapsing (a.k.a., combining) the Asian students group with the Others group (the baseline of comparison in model 1), which leads to model 2 where the new category of Others/Asian is the new baseline level. Clearly, $r_{Y\left(X_{1}, X_{2} X_{3} X_{4} X_{5}\right)}^{2}$ serves as a numeric measure of the importance of distinguishing Asian students from students in the Others group in terms of the proportion of the total variability in the DV, after adjusting for the distinction between White and non-White, the distinction between African American and non-African American, and the effects of any other predictors.

Given that $r_{Y\left(X_{1}, X_{2} X_{3} X_{4} X_{5}\right)}^{2}=1.85 \%$, we conclude that, within those students who are neither White nor African American and are identical on admission ACT score and high school GPA, a new distinction made between Asian and other students is estimated to help the model explain additional $1.85 \%$ of the total variability of the DV beyond the proportion already explained by $X_{4}$ and $X_{5}$ and the classification of students into only three groups: White, African American, and Others/Asian. Stated differently, if we stop making the distinction between Asian students and students in the Others group (as in model 2), the predictive power of our model would drop by an amount that is about $1.85 \%=\left[R_{Y . X_{1} X_{2} X_{3} X_{4} X_{5}}^{2}\right.$ $R_{Y . X_{2} X_{3} X_{4} X_{5}}^{2}$ ] of the total DV variability, given that we continue to keep in the model the distinction between White and non-White, the distinction between African American and non-African American, admission ACT score, and high school GPA. Equivalently, the model estimates that the distinction between Asian students and students in the Others group explains about $1.85 \%$ of the total DV variability.

With $r_{Y X_{1} \cdot X_{2} X_{3} X_{4} X_{5}}$ and $r_{Y\left(X_{1} \cdot X_{2} X_{3} X_{4} X_{5}\right)}$ explained, correlations for the other two dummy variables can be interpreted in a similar manner. Each time we work on a dummy variable, we need to keep constant the distinctions made in all other dummy variables and also keep constant all other predictors, if any. 
Yang et al.

\section{Partial and Semi-Partial Correlations for Other Predictors}

So far, we have examined partial and semi-partial correlations for dummy indicators created for a categorical predictor. For completeness, we next briefly interpret the two types of correlations for the other predictors when the categorical predictor is statistically controlled. We examine the correlations for $X_{4}$, admission ACT score: 1) Partial correlation $\left.r_{Y X_{4} \cdot X_{1} X_{2} X_{3} X_{5}}=0.157,2\right)$ semi-partial correlation $r_{Y\left(X_{4} \cdot X_{1} X_{2} X_{3} X_{5}\right)}=0.133$, and 3) zero-order correlation $r_{Y X_{4}}=0.357$.

After squaring $r_{Y X_{4} \cdot X_{1} X_{2} X_{3} X_{5}}$ and $r_{Y\left(X_{4} \cdot X_{1} X_{2} X_{3} X_{5}\right)}$, we obtain $r_{Y X_{4} \cdot X_{1} X_{2} X_{3} X_{5}}^{2}=0.025$ and $r_{Y\left(X_{4} \cdot X_{1} X_{2} X_{3} X_{5}\right)}^{2}=$ 0.018 . We see that $X_{4}$ uniquely explains about $1.8 \%$ of the total DV variability based on the coefficient of semi-partial determination, but $2.5 \%$ of the remaining DV variability not already explained by all other predictors according to the coefficient of partial determination.

Suppose that we are interested in the correlation between the DV (college GPA) and $X_{4}$ (admission ACT score). On the face of it, a moderate correlation (0.357) seems to exist between these two variables. However, $X_{4}$ is also moderately correlated (0.470) with $X_{5}$ (high school GPA), and weakly correlated (from 0.049 to 0.171 in absolute value) with the three dummy variables for the categorical predictor $X_{\text {Population. }}$ A research question might be, then "What would the relationship between the DV (college GPA) and $X_{4}$ (admission ACT score) have been if we examined only those students from one of the four ethnic groups who also had identical high school GPA?" Clearly, this question can be answered by the partial correlation between the DV and $X_{4}$ when controlling for all other predictors in the model. The correlation between college GPA and admission ACT score is reduced noticeably, from $r_{Y X_{4}}=0.357$ to $r_{Y X_{4} \cdot X_{1} X_{2} X_{3} X_{5}}=0.157$. On the other hand, a second research question might be, "What would the relationship between the DV (college GPA) and $X_{4}$ (admission ACT score) have been if we adjusted only admission ACT score for high school GPA and the four ethnic groups, a.k.a., if we removed high school GPA and ethnic group as two sources of variation from admission ACT score, but not from college GPA?" Clearly, this second question can be answered by the semi-partial correlation between the DV and $X_{4}$. This time, the correlation between college GPA and admission ACT score is also reduced noticeably, from $r_{Y X_{4}}=0.357$ to $r_{Y\left(X_{4} \cdot X_{1} X_{2} X_{3} X_{5}\right)}=0.133$.

It is believed that this noticeable reduction in the value of the correlation between the DV and $X_{4}$, after removing the effects of a linear combination of $X_{5}$ and $X_{\text {Population }}$ from one $\left(X_{4}\right)$ or both variables, is more related to the statistical control of $X_{5}$ (with which $X_{4}$ is moderately correlated at .470) than to that of $X_{\text {Population }}$ (with which $X_{4}$ is weakly correlated, from .049 to .171 in absolute value). To find supportive evidence for this, we run a second analysis where another model (model 3a) is estimated which contains everything that model 1 has with the exception of $X_{5}$ (high school GPA). This time, when computing the partial and semi-partial correlations between the DV and $X_{4}$, we statistically control only $X_{\text {Population }}$ but without adjusting for $X_{5}$. This time, there is hardly any reduction in the correlation between the DV and $X_{4}: r_{Y X_{4}}=0.357, r_{Y X_{4} \cdot X_{1} X_{2} X_{3}}=0.355$, and $r_{Y\left(X_{4}, X_{1} X_{2} X_{3}\right)}=0.348$, which are almost identical. By contrast, if we choose to statistically control $X_{5}$ without adjusting for $X_{\text {Population }}$ (model 3b), the noticeable reduction in the value of the correlation between the DV and $X_{4}$ is once again observed: $r_{Y X_{4}}=0.357,, r_{Y X_{4} \cdot X_{5}}=$ 0.158 , and $r_{Y\left(X_{4} \cdot X_{5}\right)}=0.137$. Finally, similar patterns of change are observed when correlating $X_{5}$ with the DV. To see the effect of statistically controlling only $X_{\text {Population }}$ on the correlation calculated for $X_{5}$ (but without adjusting for $X_{4}$ ), we run a third analysis where another model (model 4a) is estimated which contains everything that model 1 has with the exception of $X_{4}$ (admission ACT score). The observation is that the statistical control of only $X_{\text {Population }}$ hardly has any effect on the correlation between the DV and $X_{5}: r_{Y X_{5}}=0.501, r_{Y X_{5} . X_{1} X_{2} X_{3}}=0.301$, and $r_{Y\left(X_{5} . X_{1} X_{2} X_{3}\right)}=0.491$, which are almost identical. By contrast, if we choose to statistically control $X_{4}$ but without adjusting for $X_{\text {Population }}$ (model $4 \mathrm{~b}$ ), the noticeable reduction in the value of the correlation between the DV and $X_{5}$ is once again observed: $r_{Y X_{5}}=0.501$, , $r_{Y X_{5} . X_{4}}=0.405$, and $r_{Y\left(X_{5} . X_{4}\right)}=0.378$. The SPSS code for these two additional analyses (models $3 \mathrm{a}$ through $4 \mathrm{~b}$ ) is also provided in the appendix. 


\section{Evaluating Relative Importance of Predictors}

Another important topic in educational research is that of assessing relative importance of predictors. Most of the time, measuring relative importance refers to an explicit quantification of the relative contributions of individual predictors to the model's total explanatory power, and it revolves around the determination of a rank ordering of the predictors based on a criterion of choice. (Azen \& Budescu, 2003; Azen \& Traxel, 2009; Budescu, 1993; Courville \& Thompson, 2001; Darlington, 1968; Luo \& Azen, 2013; Nathans, Oswald, \& Nimon, 2012; Pedhazur, 1997; Thompson, 1997; Thompson \& Borrello, 1985). However, there is no universally accepted definition of how to quantify predictor importance, and various methods have been suggested in the literature (Achen, 1982; Bring, 1994; Kruskal \& Majors, 1989). These proposed methods are in the form of statistics or procedures measuring predictor contributions from various perspectives. Also, in the literature, there exist multiple ways of labeling these perspectives, leading to different ways of categorizing or grouping these predictor importance methods.

Following Budescu (1993) and Azen and Budescu (2003), among such methods are slope-based measures (standardized regression coefficients, etc.) and correlation-based variance decomposition measures (squared partial and semi-partial correlations, etc.). But, the list does not stop there. There are many variable importance methods which do not fall into the two categories described above. They include the relatively simple Pratt index which combines regression coefficients and correlations between predictors and the DV (Liu, Zumbo, \& Wu, 2014; Pratt, 1987; Thomas, Hughes, \& Zumbo, 1998; Zumbo, 2007) and several other mathematically challenging procedures which measure importance using effects averaged across all possible importance ordering sequences (Theil, 1987; Theil, \& Chung, 1988). Among methods of measuring predictor importance, dominance analysis (DA) is probably one of the most respected these days which have been widely investigated and implemented (Azen, \& Budescu, 2003; Azen, \& Budescu, 2006; Azen, \& Traxel, 2009; Budescu, 1993; Budescu, \& Azen, 2004; Luo, \& Azen, 2013). From a different perspective, according to Johnson and LeBreton (2004), some importance measures are single-analysis methods because they use the output from a single regression analysis: Squared partial and semi-partial correlations, Pratt index, etc. By contrast, others are multiple-analysis methods in that they combine the results from more than one regression analysis involving different combinations of the same variables: DA, methods based on averaging over orderings, etc. To sum up, the literature on predictor importance is extensive and a thorough discussion is beyond the scope of this article. Interested readers should refer to review articles and annotated bibliographies like Firth (1998), Gromping (2007), Johnson and LeBreton (2004) to begin with.

Despite the richness of relative importance methods, almost all of them are inadequate in one way or another. It is thus important for applied researchers to be aware of limitations associated with these methods in order to make the right choice of which measures to use in a particular research project. Simple single analysis methods measuring marginal contributions of individual predictors (e.g., standardized regression coefficients and semi-partial correlations) and those that do not involve marginal contributions (e.g., structure coefficients and zero-order correlations) may not agree with each other and thus paint conflicting pictures of predictor importance (an example is to be provided next). This happens even more frequently given strong collinearity among predictors and/or suppressor variables (Budescu, 1993; Cramer, 1974; Tzelgov \& Henik, 1991). On the other hand, most multi-analysis methods based on averaging over all ordering of predictors are computationally challenging, their justifications have originally been quite ad hoc and their properties are not yet well understood (Johnson \& LeBreton, 2004). At this point, dominance analysis is generally considered to be one of the most successful methods in the literature, but the procedure also suffers from computational complexity, making it difficult to implement. Again, the limitations of various importance measures are extensive and need multiple dedicated studies to cover. Here, we refer the readers to Johnson and LeBreton in which a concise discussion is provided of limitations of a full range of predictor importance statistics and procedures.

Back to partial and semi-partial correlations, they have an important role to play in assessing predictor importance (Azen \& Budescu, 2003). Kruskal (1987) advocates squared partial correlations represent importance of predictors whereas Lindeman, Merenda, and Gold (1980) propose a similar procedure based on the squared semi-partial correlations. In addition, a variant of measuring importance through partial correlations is known as commonality analysis (Mood, 1969, 1971; Newton \& Spurrell, 1967a, 1967b). Finally, in Budescu's (1993) dominance analysis, the contribution to prediction is defined as the squared semi-partial correlation. 
Yang et al.

In regression, together with partial and semi-partial correlations, several other importance statistics are commonly used simply because they are typically made available in standard regression computer programs: 1) Standardized regression coefficients, 2) statistical significance tests for regression parameters, and 3) zero-order correlation between the DV and each predictor (This correlation is mathematically equivalent to the structure coefficient for the predictor in assessing relative importance of predictors, although they are different in value (Courville \& Thompson, 2001; Pedhazur, 1997; Thompson \& Borrello, 1985)). In addition, there are also statistics software programs which focus on evaluating predictor importance and thus have many of the newer methods built-in: Gromping (2006) and Nimon, Oswald, and Roberts (2013).

As is well known, when predictors are uncorrelated with each other, many different measures of relative importance are equivalent in the sense of leading to the same rank ordering of predictors (Gromping, 2007). Unfortunately, this simple picture does not apply to multiple dummy variables from a single categorical predictor with three or more categories. These dummy variables are always correlated to some extent because they are never independent of each other (Cohen et al., 2003). So, when evaluating relative importance of dummy indicators, we have to take into account the extent to which they overlap. Therefore, statistics that are able to measure marginal contributions of individual predictors can be used with dummy predictors: Partial and semi-partial correlations, standardized regression coefficients, observed level of significance ( $\mathrm{p}$-value) from partial t-tests on regression parameters, etc. Next, we examine the relative importance of dummy indicators for the categorical predictor $X_{\text {Population }}$ using the above partial statistics that are able to partial out the effects from other aspects of the model on the DV and measure marginal contributions of each individual predictor. We also compare the results with those from using zero-order correlations which do not have the partial-out capability.

From Table 1, the results on the rank ordering of predictors are mixed, and depend on if the criterion is one of the partial statistics or the zero-order correlation. With the exception of $X_{5}$ which all five criteria agree contributes most to the prediction of the DV, the results of ranking of relative importance of the three dummy indicators are in two different groups:

1. Group one: Dum1_Asian > Dum3_AfricanAmerican > Dum2_White, as suggested by standardized coefficients, values of observed level of significance ( $p$-value), partial and semipartial correlations

2. Group two: Dum2_White > Dum3_AfricanAmerican > Dum1_Asian, as suggested by zeroorder correlations

Among the five criteria used here, four of them are compared in terms of their absolute values and are in higher-is-better (more important) format, whereas only the p-values are evaluated without taking the absolute value and they are in lower-is-better (more important) format. That said, we should note that the zero-order correlation statistic utilized in group two may be inappropriate to use because this particular statistic is unable to factor in the overlap between the three dummy indicators that belong to the same categorical predictor. The failure to do so may lead to erroneous conclusions because zero-order correlations and partial/semi-partial correlations may give us very different pictures of the interrelationships among those variables (Cohen et al., 2003; Hays, 1994; IBM SPSS, 2012). Therefore, it is the group one results that we primarily count on during the evaluation process because they are more statistically reasonable. We also recommend that the results be further assessed under some other wellsupported methods from the literature: dominance analysis, etc.

In group one, all four partial criteria used are consistent with each other in terms of the relative importance of dummy indicators. First, the distinction between Asian students and students in the Others group contributes the most to the prediction of college GPA under the existing way of classifying students (Asian, White, African American, and Others), after holding constant the effects of admission ACT score and high school GPA. In other words, when examining students who are identical on admission ACT score and high school GPA, the model estimates that Asian students are significantly lower than students in the Others group in terms of college GPA. Second, the extent is less between African American students and students in the Others group, although the pattern is similar. Given identical admission ACT score and high school GPA, African American students are not as effective as students in the Others 
group in earning high college GPA. As for White students, there is some evidence that they tend to be lower on college GPA than students in the Others group, but the evidence is marginal at best. Additionally, we note that Dum2_White has the least significant p-value (0.530) among all three dummy variables, but its zero-order correlation in group two indicates the strongest correlation (0.148) with the DV. So, although the p-value indicates that Dum2_White is the least useful dummy indicator out of the three, its zero-order correlation with the DV suggests just the opposite. To interpret this conflicting scenario, we also examine its partial (-0.042) and semi-partial (-0.035) correlations, each one being the weakest among the three dummy variables. Clearly, partial and semi-partial correlations agree with the pvalue in terms of the relative importance of this dummy indicator. This may not be surprising because partial statistics are able to measure the marginal contribution of the individual predictor to the model after adjusting for the effects of all other predictors, whereas the zero-order correlation does not have such a capability. Also, this case with Dum2_White is a typical demonstration of how converting a zero-order Pearson correlation to a partial/semi-partial correlation may change our impression of observed relationship between variables (Hays, 1994; IBM SPSS, 2012). Given that the zero-order correlation between Dum2_White and the DV is .148, we say that the correlation between college GPA and a variable that distinguishes White students from non-White students may be small at about 0.148. But, when we remove from all non-White students those who are either Asian or African American students (by factoring in the other two dummy indicators), the observed correlation between Dum2_White and the DV almost completely diminishes, suggesting that there is hardly any difference between White students and students in the Others group in terms of college GPA.

\section{Conclusion}

This article discusses partial and semi-partial correlations for a categorical predictor in linear regression to undo two misconceptions about these correlations. To address misconception 1, the article demonstrates the concept of partial correlation is applicable to a categorical predictor variable given that the predictor is properly coded into one or more dummy variables. For each dummy variable, its partial correlation represents the corresponding unstandardized regression parameter estimate in a correlational rather than raw score metric. The partial correlation serves as an expression in correlational terms of the difference between the indicated group and the reference group in terms of the DV scores. To address misconception 2, the article interprets the squared semi-partial correlation for a dummy variable as representing the reduction in the multiple coefficient of determination $\left(R^{2}\right.$ statistic) when the category indicated by this variable is collapsed or combined with the baseline category of comparison. Stated differently, the squared semi-partial correlation serves as a measure, in terms of the proportion of total DV variability, of the importance of distinguishing the group indicated by this variable from the baseline level. Finally, this article examines the two correlations under one of their major areas of application: Evaluating predictor importance. Conceptually, it is generally agreed that measuring predictor importance involves quantification of the relative contributions of individual predictors to the model's total explanatory power. In practice, there exist many different ways of quantifying the contributions of an individual predictor and grouping those relative importance methods. The article briefly mentions many such methods, alludes to their inadequacies, and offers many recommended readings and resources that interested readers can consult about understanding and implementing these methods. The article emphasizes that methods able to partial out effects of other predictors when assessing predictor importance often disagree with those that do not have the capability, thus creating a conflicting picture of relative importance of predictors. In the numerical example, the article demonstrates a case of this discrepancy. Although this article favors results from methods with the partial-out capability, it is recommended to subject the results to further analysis under dominance analysis and other well-supported methods from the literature.

\section{References}

Achen, C. H. (1982). Interpreting and using regression. Beverly Hills, CA: Sage Publications.

Ary, D., Jacobs, L. C., Sorensen, C. K., Walker, D. (2013). Introduction to research in education (9th ed.). Independence, KY: Cengage Learning.

Azen, R., \& Budescu, D. V. (2003). The dominance analysis approach for comparing predictors in multiple regression. Psychological Methods, 8(2), 129-148. 
Yang et al.

Azen, R., \& Budescu, D. V. (2006). Comparing predictors in multivariate regression models: An extension of dominance analysis. Journal of Educational and Behavioral Statistics, 31(2), 157-180.

Azen, R., \& Traxel, N. M. (2009). Using dominance analysis to determine predictor importance in logistic regression. Journal of Educational and Behavioral Statistics, 34, 319-347.

Bring, J. (1994). How to standardize regression coefficients. American Statistician, 48, 209-213.

Budescu, D. V. (1993). Dominance analysis: A new approach to the problem of relative importance of predictors in multiple regression. Psychological Bulletin, 114, 542-551.

Budescu, D. V., \& Azen, R. (2004). Beyond global measures of relative importance: Some insights from dominance analysis. Organizational Research Methods, 7(3), 341-350.

Cohen, J., Cohen, P., West, S. G., \& Aiken, L. S. (2003). Applied multiple regression/correlation analysis for the behavioral sciences (3rd ed.). Mahwah, NJ: Lawrence Erlbaum Associates, Inc.

Courville, T., \& Thompson, B. (2001). Use of structure coefficients in published multiple regression article: $\beta$ is not enough. Educational and Psychological Measurement, 61(2), 229-248.

Cramer, E. M. (1974). Brief report: The use of highly correlated predictors in regression analysis. Multivariate Behavioral Research, 9(2), 241-243.

Darlington, R. B. (1968). Multiple regression in psychological research and practice. Psychological Bulletin, 69(3), 161-182.

Draper, N. R., \& Smith, H. (1981). Applied regression analysis (2nd ed.). New York: John Wiley \& Sons, Inc.

Edwards, A. L. (1976). An introduction to linear regression and correlation. San Francisco: W. H. Freeman and Company.

Field, A. (2009). Discovering statistics using SPSS (3rd ed.). Thousand Oaks, CA: Sage.

Firth, D. (1998). Relative importance of explanatory variables: An annotated bibliography. Retrieved from http://warwick.ac.uk/dfirth/papers/Stockholm1998.pdf

Gelman, A., \& Hill, J. (2007). Data analysis using regression and multilevel/hierarchical models. New York: Cambridge University Press.

Gromping, U. (2006). Relative importance for linear regression in R: The package relaimpo. Journal of Statistical Software, 17(1), 1-27.

Gromping, U. (2007). Estimators of relative importance in linear regression based on variance decomposition. The American Statistician, 61(2), 139-147.

Hays, W. (1994). Statistics (5th ed.). Stamford, CT: Cengage Learning.

IBM SPSS (2012). IBM SPSS Statistics Base 21. Chicago: Author.

Johnson, B., \& Christensen, L. (2010). Educational research: Quantitative, qualitative, and mixed approaches (4th ed.). Thousand Oaks, CA: Sage.

Johnson, J. W., \& LeBreton, J. M. (2004). History and use of relative importance indices in organizational research. Organizational Research Methods, 7(3), 238-257.

Keppel, G. (1991). Design and analysis: A researcher's handbook (3rd ed.). Englewood Cliffs, NJ: Prentice Hall.

Kruskal, W. (1987). Relative importance by averaging over orderings. The American Statistician, 41, 610.

Kruskal, W., \& Majors, R. (1989). Concepts of relative importance in recent scientific literature. The American Statistician, 43, 2-6.

Kutner, M. H., Nachtsheim, C. J., Neter, J., \& Li, W. (2005). Applied linear statistical models (5th ed.). New York: McGraw-Hill/Irwin.

Lewis-Beck, M. S. (1980). Applied regression: An introduction. Beverly Hills, CA: Sage Publications, Ltd.

Ley, P. (1972). Quantitative aspects of psychological assessment. London: Gerald Duckworth and Company.

Lindeman, R. H., Merenda, P. F., \& Gold, R. Z. (1980). Introduction to bivariate and multivariate Analysis. Glenview, IL: Scott Foresman.

Liu, Y., Zumbo, B. D., \& Wu, A. D. (2014). Relative importance of predictors in multilevel modeling. Journal of Modern Applied Statistical Methods, 13(1), 2-22. 
Luo, W., \& Azen, R. (2013). Determining predictor importance in hierarchical linear models using dominance analysis. Journal of Educational and Behavioral Statistics, 38(1), 3-31.

Martella, R. C., Nelson, J. R., Morgan, R. L., \& Marchand-Martella, N. E. (2013). Understanding and interpreting educational research. NYC, NY: The Guilford Press.

Mendenhall, W., \& Sincich, T. (2003). A second course in statistics: Regression analysis (6th ed.). Upper Saddle River, NJ: Pearson Education, Inc.

Monahan, J. F. (2008). A primer on linear models. Boca Raton, FL: Chapman \& Hall/CRC.

Montgomery, D. C., Peck, E. A., \& Vining, G. G. (2001). Introduction to linear regression analysis (3rd ed.). New York: John Wiley \& Sons, Inc.

Mood, A. M. (1969). Macro-analysis of the American educational system. Operations Research, 17, 770784.

Mood, A. M. (1971). Partitioning variance in multiple regression analyses as a tool for developing learning models. American Educational Research Journal, 8, 191-202.

Muller, K. E., \& Fetterman, B. A. (2002). Regression and ANOVA: An integrated approach using SAS software. Cary, NC: USA.

Nathans, L. L., Oswald, F. L., \& Nimon, K. (2012). Interpreting multiple linear regression: A guidebook of variable importance. Practical Assessment, Research \& Evaluation, 17(9), 1-19.

Neter, J., \& Wasserman, W. (1974). Applied linear statistical models: Regression, analysis of variance, and experimental design. Homewood, IL: Richard D. Irwin, Inc.

Newton, R. G., \& Spurrell, D. J. (1967a). A development of multiple regression for the analysis of routine data. Applied Statistics, 16, 51-64.

Newton, R. G., \& Spurrell, D. J. (1967b). Examples of the use of elements for clarifying regression analysis. Applied Statistics, 16, 165-172.

Nimon, K., Oswald, F. \& Roberts, J. K. (2013). yhat: Interpreting regression effects [Computer software manual]. https://CRAN.R-project.org/package=yhat. (R package version 2.0.0.)

Pedhazur, E. J. (1997). Multiple regression in behavioral research (3rd ed.). Fort Worth, TX: Harcourt Brace.

Pedhazur, E. J., \& Schmelkin, L. P. (1991). Measurement, design, and analysis: An integrated approach. New York: Psychology Press.

Phelps, S. (1923). How partial and multiple correlations can be used by the school administrator. Peabody Journal of Education, 1(3), 149-159.

Pratt, J. W. (1987). Dividing the indivisible: Using simple symmetry to partition variance explained. In T. Pukilla \& S. Duntaneu (Eds.), Proceedings of second Tampere conference in statistics (pp. 245-260). University of Tampere, Finland.

$\begin{array}{lllll}\text { Price, } & \text { J. } & \text { (n.d.). } & \text { Partial forrelation. }\end{array}$ http://people.uncw.edu/pricej/teaching/statistics/partial_correlation.htm

Psychwiki. (n.d.). What is a partial correlation? Retrieved from http://www.psychwiki.com/wiki/What_is_a_partial_correlation\%3F

Rao, C. R., \& Toutenburg, H. (1999). Linear models: Least squares and alternatives (2nd ed.). New York: Springer-Verlag.

Sachar, J. (1980). Cautions in the interpretation of the partial correlation coefficient. The Journal of Experimental Education, 48(3), 209-216.

Searle, S. R. (1971). Linear models. New York: John Wiley \& Sons, Inc.

Seber, G. A. F., \& Lee, A. J. (2003). Linear regression analysis (2nd ed.). Hoboken, NJ: John Wiley \& Sons, Inc.

Tamhane, A. C., \& Dunlop, D. D. (2000). Statistics and data analysis: From elementary to intermediate. Upper Saddle River, NJ: Prentice-Hall, Inc.

Theil, H. (1987). How many bits of information does an independent variable yield in a multiple regression? Statistics \& Probability Letters, 6, 107-108.

Theil, H., \& Chung, C.-F. (1988). Information-theoretic measures of fit for univariate and multivariate linear regressions. The American Statistician, 42, 249-252.

Thomas, D. R., Hughes, E., \& Zumbo, B. D. (1998).On variable importance in linear regression. Social Indicators Research, 45, 253-275. 
Yang et al.

Thompson, B. (1997). Variable importance in multiple regression and canonical correlation. In B. Thompson (Ed.), Advances in social science methodology, volume 4 (pp. 107-136). Bingley, UK: Emerald Group Publishing Limited.

Thompson, B., \& Borrello, G. M. (1985). The importance of structure coefficients in regression research. Educational and Psychological Measurement, 45(2), 203-209.

Tzelgov, J., \& Henik, A. (1991). Suppression situations in psychological research: Definitions, implications and applications. Psychological Bulletin, 109, 524-536.

Weisberg, S. (2005). Applied linear regression (2nd ed.). Hoboken, NJ: John Wiley \& Sons, Inc.

Yan, X., \& Su, X. G. (2009). Linear regression analysis: Theory and computing. Singapore: World Scientific.

Zumbo, B. D. (2007). Validity: Foundational issues and statistical methodology. In C. R. Rao and S. Sinharay (Eds.), Handbook of statistics, volume 26: Psychometrics (pp. 45-79). The Netherlands: Elsevier Science.

Send correspondence to: $\quad$ Hongwei Yang

Arizona State University

Email: Patrick.Yang@asu.edu

\section{APPENDIX}

\section{Data Summary Matrix and SPSS Code}

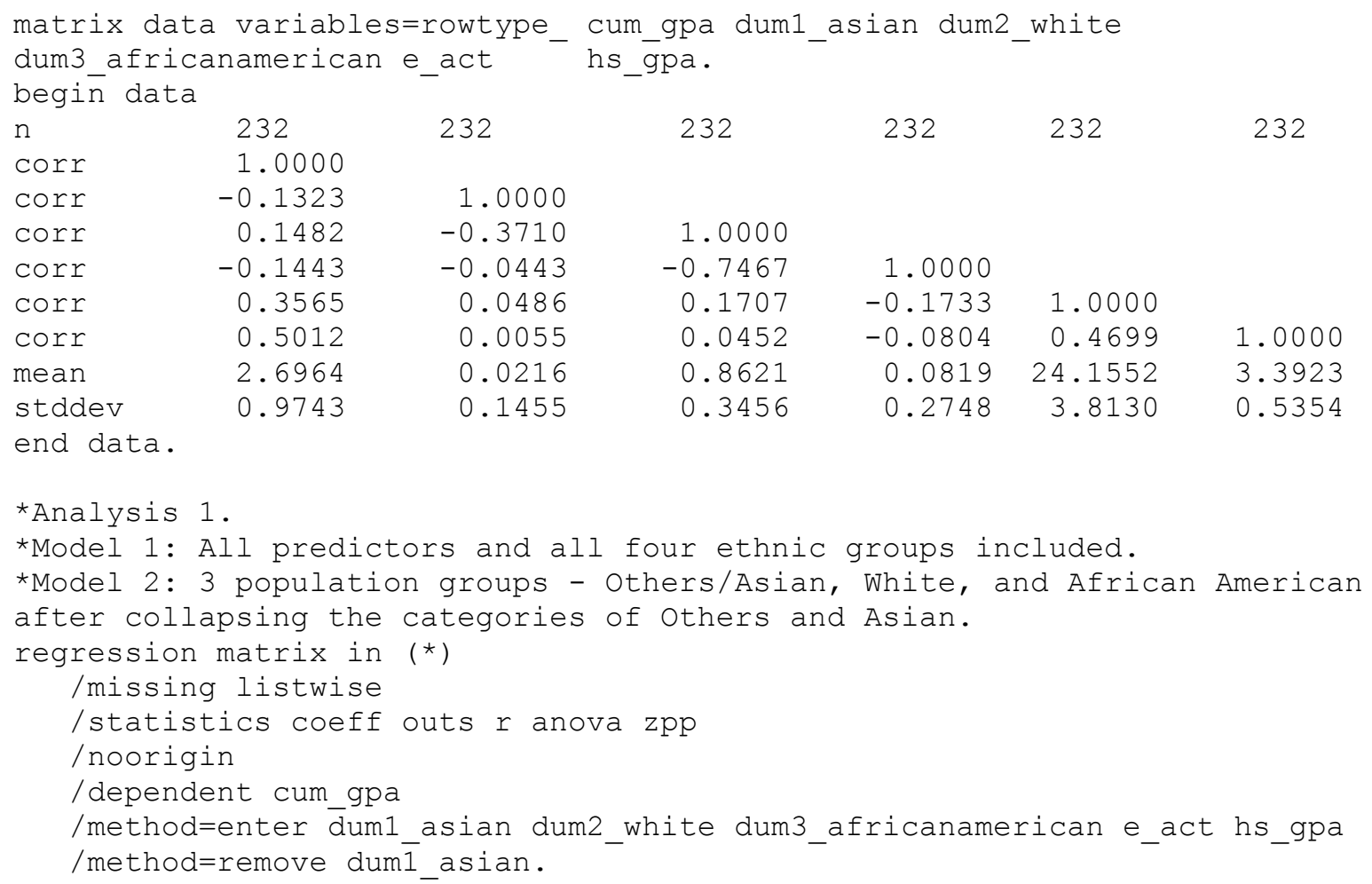




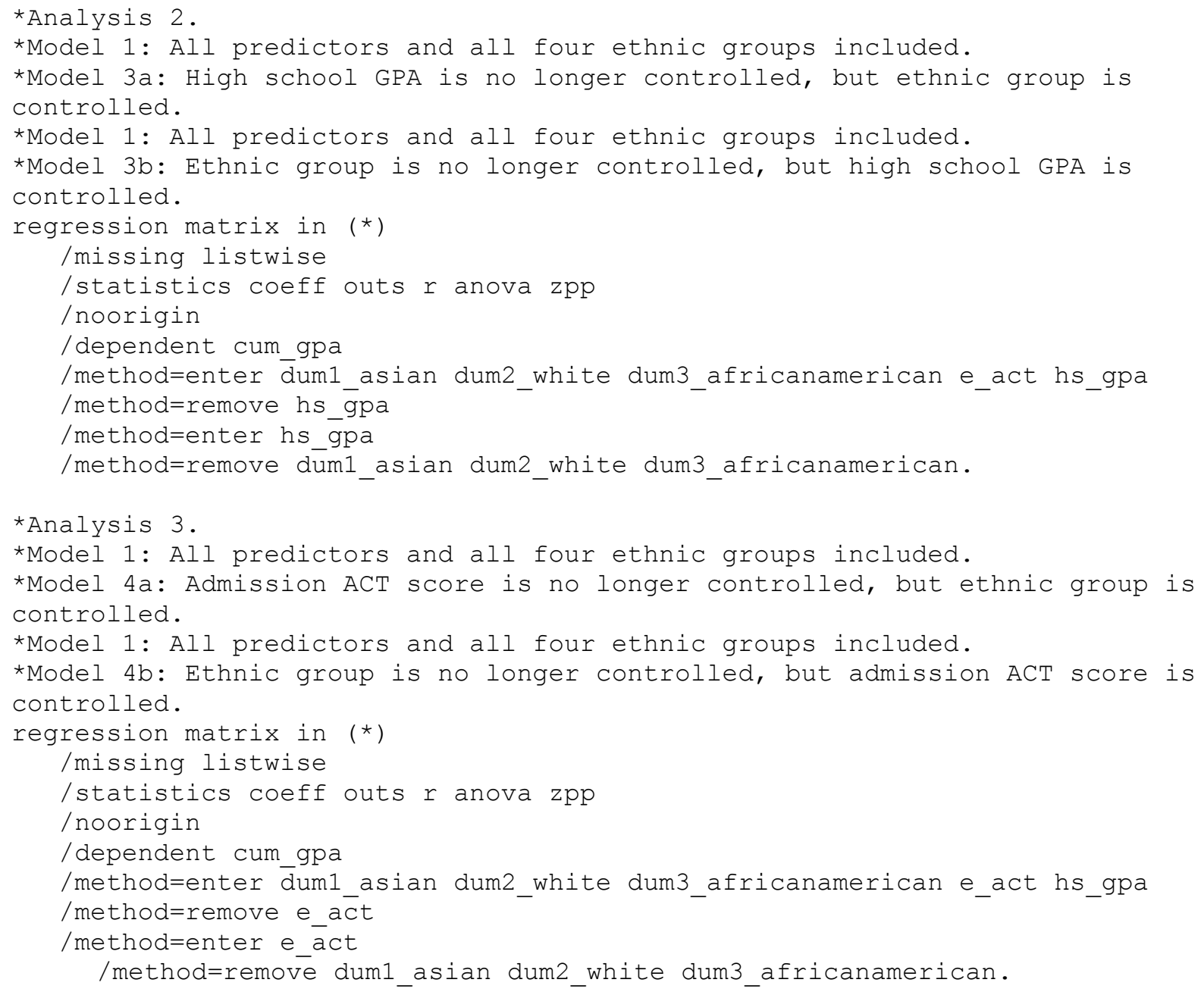

